\title{
All, Most, Some Differentiable Dynamical Systems
}

\author{
Michael Shub*
}

\begin{abstract}
In the first part of this paper we study dynamical systems from the point of view of algebraic topology. What features of all dynamical systems are reflected by their actions on the homology of the phase space? In the second part we study recent progress on the conjecture that most partially hyperbolic dynamical systems which preserve a smooth invariant measure are ergodic, and we survey the known examples. Then we speculate on ways these results may be extended to the statistical study of more general dynamical systems. Finally, in the third part, we study two special classes of dynamical systems, the structurally stable and the affine. In the first case we study the relation of structural stability to entropy, and in the second we study stable ergodicity in the homogeneous space context.
\end{abstract}

Mathematics Subject Classification (2000). Primary 37 Secondary 37C,37D.

Keywords. dynamical system, entropy, Entropy Conjecture, partial hyperbolicity, accessibility, ergodicity, Lyapunov exponent, SRB measure, structural stability, affine diffeomorphism.

\section{Introduction}

We study discrete differentiable dynamical systems $f: M \rightarrow M$ on a smooth closed manifold of dimension $m$. ${ }^{*}$ Thus, $f \in \operatorname{Diff}^{r}(M)$ or $\operatorname{End}^{r}(M)$, the $C^{r}$ diffeomorphisms or endomorphisms of $M$ respectively, where $1 \leq r \leq \infty$, and occasionally, $r=0$.

What can be said about differentiable dynamical systems? The best things that can be said concern all systems. When we can't make statements about all systems we may content ourselves with most systems. We expect that properties which hold for most systems hold for a specific system under consideration, but we can't be sure until we've proven it.

Section 2 concerns properties which may hold for all dynamical systems, mainly properties from algebraic topology. Principal among these is the Entropy Conjecture which relates the topological entropy of a dynamical system to the induced map on the homology groups.

\footnotetext{
* The author would like to thank Charles Pugh for years of collaboration and also for help in preparing this article.

* "Closed" means that $M$ is compact and has empty boundary.
} 
In section 3 we turn from all to most. We investigate the time honored role of (a) some hyperbolicity, especially as it concerns (b) the stable and unstable manifolds of points, (c) their intersections and (d) the equivalence relation these intersections define in the manifold. In the by now classical uniformly hyperbolic case, the equivalence classes form Smale's spectral decomposition and the behavioral properties entailed are structural stability, SRB measures, and ergodicity in the volume preserving Anosov case.

Uniformly hyperbolic systems are some, not most dynamical systems. So from the point of view of hoping to describe most dynamical systems we relax the structural properties to some hyperbolicity . Our goal is to understand how hypotheses about (a)-(d) affect ergodicity of volume preserving diffeomorphisms and whether these hypotheses hold for most partially hyperbolic volume preserving diffeomorphisms. Later we speculate on how they may affect the existence of SRB measures. Our theme is that a little hyperbolicity goes a long way toward ergodicity. Part of our problem is that the (un)stable manifolds, their intersections, and the equivalences they define are topological objects, while the desired results we wish to conclude are measure theoretic. Working in mixed categories raises rather severe technical difficulties, some of which have only recently been overcome.

We conjecture that most volume preserving partially hyperbolic dynamical systems (initially studied by Brin and Pesin) are ergodic, and we survey the rather substantial recent results in this direction, especially by Keith Burns and Amie Wilkinson, and Federico and Jana Hertz and Raul Ures. Here we first confront the role of the equivalence relation on $M$ induced by the strong stable and unstable manifolds and their intersections. This equivalence relation divides the manifold into accessibility classes. The main problem is to understand the relationship of the topologically defined accessibility classes of a partially hyperbolic dynamical system to the measure theoretically defined ergodic components via the Anosov-Hopf argument for ergodicity.

In section 4.1 we study flows on homogeneous spaces and more generally affine diffeomorphisms. The ergodic theory of affine diffeomorphisms and flows on homogeneous spaces is extremely well developed. It relies to a large extent on the structure of Lie groups and representation theory. The ergodicity results in section 3 apply outside of the homogeneous space context and per force use different techniques such as the accessibility relationship and julienne quasi-conformality. Juliennes are dynamically defined sets and quasi-conformality applies to the holonomy maps of the invariant stable and unstable manifolds. How good are these techniques when applied back in the homogeneous space context where a more elaborate set of tools is available for the study of ergodicity and stable ergodicity? While the proofs are very different there is a remarkable coincidence between those affine diffeomorphisms which are stably ergodic when considered with respect to affine perturbations and those which are stably ergodic with respect to all perturbations. Some rather interesting cases remain unresolved. The coincidence of results makes us feel that we have landed in the right place with our definitions of accessibility and makes the outstanding cases even more interesting.

In section 4.2 we see how the results of section 2 and 3 relate to one another. The 
SRB measures were initially proven to exist for uniformly hyperbolic dynamical systems. The Entropy Conjecture holds for these diffeomorphisms and we consider how sharp it is. How much complexity must a diffeomorphism have beyond that which is forced by the Entropy Inequality? Of particular interest are the MorseSmale diffeomorphisms. The study of these diffeomorphisms has a deep connection to the theory of the structure of manifolds in high dimensions accomplished by Smale. Yet there are new invariants and obstructions.

The relations between dynamics and algebraic topology studied in sections 2 and 4.2 may hold for all $r \geq 1$ but there are definite distinctions between the ergodic theory of $C^{1}$ and $C^{2}$ dynamical systems, so in sections 3 and 4.1 we mostly assume that $r \geq 2$. Sections 2 and 4.2 and sections 3 and 4.1 may be read independently of one another. But I think it would be a mistake to disassociate them. For one thing, the hyperbolic systems are partially hyperbolic. To understand the partially hyperbolic we must first understand the hyperbolic. For another, the variational principle ties measure theoretic entropy to topological entropy. (See for example Problem 3 of section 2.) One of the main themes of this talk are the structures that link and the ties that bond the topological and measure theoretic in the presence of smoothness and some hyperbolicity. Moreover, what is true for all must be taken into consideration when studying most.

\section{All Differentiable Dynamical Systems}

What dynamical properties hold for all dynamical systems $f$ ? The answer often depends on the degree of differentiability of $f$.

- Every continuous dynamical system supports an invariant probability measure.

- Every Lipschitz dynamical system has finite topological entropy, but nonLipschitz systems can have infinite topological entropy.

- Every $C^{\infty}$ dynamical system satisfies the Entropy Inequality explained below, but this can fail for Lipschitz dynamical systems that are not continuously differentiable.

Let us recall the concept of entropy and the statement of the Entropy Conjecture. The topological entropy of $f$ measures the growth rate of its epsilon distinguishable orbits. It makes sense for any continuous endomorphism of a compact metric space, $f: X \rightarrow X$. Given $\epsilon>0$ and $n \in \mathbb{N}$, let $N(f, n, \epsilon)$ be the maximum cardinality of a subset $A \subset X$ such that for each pair of distinct points $x, y \in A$ there is an iterate $f^{j}$ with $0 \leq j \leq k$ and

$$
d\left(f^{j}(x), f^{j}(y)\right)>\epsilon .
$$

Then, $h(f, \epsilon)$ is the exponential growth rate of $h(f, n, \epsilon)$ as $n \rightarrow \infty$, namely

$$
h(f, \epsilon)=\limsup _{n \rightarrow \infty} \frac{1}{n} \ln h(f, n, \epsilon) .
$$


The supremum of $h(f, \epsilon)$ over all $\epsilon>0$, or what is the same thing, its limit as $\epsilon \rightarrow 0$, is the topological entropy of $f, h(f)$. In [New1], Newhouse surveys how the concept of entropy fits into the $C^{r}$ category.

There is a corresponding growth rate in algebraic topology. The map $f: M \rightarrow$ $M$ induces a homology homomorphism $f_{*}: H_{*}(M, \mathbb{R}) \rightarrow H_{*}(M, \mathbb{R})$. Under $f_{*}^{n}$, homology classes grow no more rapidly than $s^{n}$ where $s=s\left(f_{*}\right)$ is the spectral radius of $f_{*}$, i.e., the modulus of the largest eigenvalue of $f_{*_{i}}: H_{i}(M, \mathbb{R}) \rightarrow$ $H_{i}(M, \mathbb{R}), 0 \leq i \leq m$.

Conjecture (Entropy Conjecture). [Sh2] For all $C^{r}$ dynamical systems, $r \geq 1$, we have the Entropy Inequality

$$
h(f) \geq s\left(f_{*}\right) .
$$

Of course, the conjecture for $r=1$ implies all the others, so this is the principal case. But if it fails for $r=1$ and holds for larger $r$, this is also interesting. The Entropy Conjecture is true for $C^{\infty}$ dynamics, but remains unknown for $C^{r}$ dynamics, $1 \leq r<\infty$. The positive result is due to Yomdin, [Yom], who compares the growth rate of the volumes of submanifolds of $M$ under iteration of $f$ to the entropy. See also [Gro2].

The Entropy Conjecture is in general false for Lipschitz endomorphisms already on the 2-sphere, and also for Lipschitz or piecewise linear homeomorphisms in dimension four or larger, $[\mathrm{Pu}]$. For $C^{1} f$, Misiurewicz and Przytycki [MiPr] prove that $h(f) \geq \ln \left(\operatorname{degree}\left(f_{* m}\right)\right)$. Some entropy lower bounds are known for continuous endomorphisms in terms of the growth rate of the induced map on the fundamental or first homology group, [Ma1, Bo, FaSh]. These imply entropy lower bounds for homeomorphisms of manifolds below dimension 4 by Poincaré duality. See [MaPr] for recent results.

Here are some more problems which are of a similar nature, relating algebraic topology to differentiable dynamics. We use the notation

$$
\operatorname{GR}\left(a_{n}\right)=\limsup _{n \rightarrow \infty} \frac{1}{n} \ln a_{n}
$$

to denote the exponential growth rate of a sequence $\left(a_{n}\right)$ in $(0, \infty]$.

Let $V$ and $W$ be closed smooth submanifolds of complementary dimension in the closed manifold $M$, and let $f$ be a smooth endomorphism of $M$. Let $N_{n}$ denote the number of distinct points of intersection of $f^{n}(V)$ with $W$ and let $I_{n}$ denote the intersection of the homology classes $f_{*}^{n}[V]$ and $[W]$, where $[V]$ and $[W]$ are the homology classes in $M$ represented by $V$ and $W$.

Problem (1). Is $\operatorname{GR}\left(N_{n}\right) \geq \operatorname{GR}\left(I_{n}\right)$ ?

A special case of this problem concerns the Lefschetz formula. Let $N_{n}(f)$ be the number of geometrically distinct periodic points of $f$ of period $n$. Let $L\left(f^{n}\right)=$ $\sum_{i=0}^{m}(-1)^{i} \operatorname{trace}\left(f_{* i}: H_{i}(M) \rightarrow H_{i}(M)\right)$.

Problem (2). Is $\operatorname{GR}\left(N_{n}(f)\right) \geq \operatorname{GR}\left(\left|L\left(f^{n}\right)\right|\right)$ ? 
By the transversality theorem the inequalities in the last two problems hold $C^{r}$ generically. The question is: Do they always hold? It is known that if $f$ is $C^{1}$ and $L\left(f^{n}\right)$ is unbounded then so is $N_{n}(f)$ [ShSu1]. This fails for Lipschitz maps.

A first interesting case is a smooth degree two map, $f$, of the 2-sphere. Let $N_{n}$ be the number of distinct periodic points of $f$ of period $n$.

Problem (3). Is $\operatorname{GR}\left(N_{n}\right) \geq \ln 2$ ?

The results of [MiPr] concerning topological entropy and degree and of Katok [Ka] comparing $\operatorname{GR}\left(N_{n}\right)$ to topological entropy for diffeomorphisms in dimension 2 make a start on this problem.

All these examples fall into the following general framework. Let $F$ be a functor from the category of manifolds to another category. Since a dynamical system $f$ may be iterated so may $F(f)$. We ask to compare the asymptotic behavior of the iterates of $F(f)$ and $f$. Here, we considered the functors of algebraic topology. Later the structures we consider and questions we ask for most or some $f$ consider functors such as the tangent bundle, measures, the de Rham complex, etc.

\section{Most Differentiable Dynamical Systems}

Since the range of dynamical behavior exhibited by all dynamical systems seems too large to admit a meaningful universal description applicable to all systems, many attempts have been made to describe features of most dynamical systems. SRB measures were introduced by Sinai, Ruelle and Bowen in the 1970's in the study of uniformly hyperbolic dynamical systems. The space integrals for continuous functions with respect to these measures predict the time averages of almost every Lebesgue point in the manifold. It is a fundamental result of Sinai, Ruelle and Bowen [Si, Ru1, BoRu] that a finite number of SRB measures exist for $C^{2}$ hyperbolic dynamics (technically Smale's Axiom A and no cycle systems.) Ruelle [Ru2] suggested that these measures apply much more generally. Much effort in dynamical systems in recent years has focused on Ruelle's suggestion. One widespread optimistic program dating from the late 1970s suggests that most systems have a finite (or perhaps countable) collection of ergodic SRB measures. For volume preserving diffeomorphisms of closed manifolds this program can not be correct because the KAM phenomenon insures the robust existence of positive measure sets of codimension one tori with quasi-periodic motions [ChSu, Yoc, Xi]. These tori have no non-zero Lyapunov exponents. So the existence of some non-zero exponents may be decisive for the program.

3.1. Partially hyperbolic diffeomorphisms. In contrast, we have suggested that a little hyperbolicity goes a long way towards ergodicity of volume preserving diffeomorphisms and hence (trivially) a unique SRB measure. Concretely our principal results are limited to $C^{2}$ partially hyperbolic volume preserving diffeomorphisms. These systems are generalizations of Anosov (globally hyperbolic) dynamical systems. In the Anosov case volume preserving $C^{2}$ diffeomorphisms 
are proved to be ergodic [An, AnSi], [Ho]. Brin and Pesin [BrPe] studied ergodicity of partially hyperbolic diffeomorphism with an accessibility property. The hypotheses of their ergodicity theorem were too limiting to be broadly applicable. In fact they probably almost never hold, [ShWi2], [HiPe]. In a series of papers [GrPuSh, Wi1, PuSh3, PuSh4, PuSh5, BuWi2, BuWi3, RHRHUr] these hypotheses have been replaced by ones quite generally applicable.

More precisely:

Definition. A diffeomorphism $f: M \rightarrow M$ is partially hyperbolic if there is a continuous $T f$-invariant splitting $T M=E^{u} \oplus E^{c} \oplus E^{s}$ such that $T f$ is hyperbolic on $E^{u} \oplus E^{s}$ and the hyperbolicity dominates $T f$ on $E^{c}$ in the sense that for some $\tau, \lambda$ with $1 \leq \tau<\lambda$ and positive constants $c, C$ we have

(a) For all $v \in E^{u}$ and all $n \geq 0, c \lambda^{n}|v| \leq\left|T f^{n}(v)\right|$.

(b) For all $v \in E^{s}$ and all $n \geq 0,\left|T f^{n}(v)\right| \leq C \lambda^{-n}|v|$.

(c) For all $v \in E^{c}$ and all $n \geq 0, c \tau^{-n}|v| \leq\left|T f^{n}(v)\right| \leq C \tau^{n}|v|$.

(d) The bundles $E^{u}, E^{s}$ are non-zero.

Condition (d) is present to avoid triviality. Without it, every diffeomorphism would be partially hyperbolic, for we could take $E^{c}$ as $T M$. Sometimes, one only requires $E^{u} \oplus E^{s} \neq 0$, but for simplicity we use the stronger assumption (d) in this paper.

Partial hyperbolicity means that under $T f^{n}$, vectors in $E^{c}$ grow or shrink more gradually than do vectors in $E^{u}$ and $E^{s}$. The center vectors behave in a relatively neutral fashion. The definition can be recast in several different ways. For instance, expansion of $E^{u}$ under positive iteration of $T f$ can be replaced by contraction under negative iteration. Also, non-symmetric rates can be used for expansion and contraction. More significantly, one could permit pointwise domination instead of the absolute domination as above. See [Puj, BoDíVi] for a discussion of dominated splitting. All of these refinements to the notion of partial hyperbolicity are exploited by Burns and Wilkinson in their result discussed below.

Given a smooth manifold $M$, fix a smooth volume $\mu$ on $M$. Then we say $f$ is volume preserving if it preserves this volume and we write the set of $\mu$ preserving $C^{r}$ diffeomorphisms of $M$ as $\operatorname{Diff}_{\mu}^{r}(M)$.

A diffeomorphism is ergodic if it preserves a measure and each measurable invariant set is a zero set or the complement of a zero set. No measurable invariant set has intermediate measure. Ergodicity is stable if it persists under perturbation of the dynamical system. Towards our theme that a little hyperbolicity goes a long way toward ergodicity and more optimistically toward the goal of finding SRB measures, we have our main conjecture.

Main Conjecture. Among the volume preserving $C^{r}$ partially hyperbolic dynamical systems for $r \geq 2$, the stably ergodic ones form an open and dense set.

An approach to the Main Conjecture via two additional conjectures consists in generalizing the Anosov-Hopf proof of the ergodicity of Anosov systems $\left(E^{c}=\{0\}\right)$ 
by studying the accessibility relationship. The Anosov-Hopf argument proceeds as follows. If $x, y$ are forward asymptotic then the time average of continuous functions along the orbit of $x$ equals the time average along the orbit of $y$. Reversing time, the same is true for $f^{-1}$ and points $x, y$ which are asymptotic in negative time. Now the Birkhoff ergodic theorem says that positive time averages equal negative time averages almost everywhere. So we say $x \sim y$ if $x$ and $y$ are positive or negative asymptotic and extend $\sim$ to an equivalence relation on $M$. In principle by the Birkhoff theorem time averages should be constant on equivalence classes and we may prove ergodicity by proving that the equivalence classes are measure zero or one. There are severe technical difficulties to this program but it can be made to work in the Anosov and the partially hyperbolic cases with some extra hypotheses. We say $x, y \in M$ are us-accessible if there is a piecewise differentiable path joining $x$ to $y$ and tangent either to $E^{u}$ or $E^{s}$ at every point of differentiability. A diffeomophism is e-(ssentially) accessible (in the measure theoretic sense) if the only subsets of $M$ saturated with respect to us-accessibility have measure 0 or 1. A diffeomorphism is us-accesssible if $M$ itself is a us-accessibility class. usaccessibility obviously implies e-accessibility. ${ }^{\dagger}$

Conjecture (A). Every $C^{2}$ volume preserving e-accessible partially hyperbolic diffeomorphism is ergodic.

Conjecture(B). The partially hyperbolic diffeomorphisms with the us-accessibility property are open and dense in the $C^{r}$ partially hyperbolic diffeomorphisms for every $r \geq 1$, volume preserving or not.

Conjectures A and B obviously imply the main conjecture.

Conjecture A was proven with two technical hypotheses in [PuSh4], center bunching and dynamical coherence. Burns and Wilkinson [BuWi2, BuWi3] have since removed the dynamical coherence hypothesis and improved the center bunching condition. The center bunching condition puts bounds on the ratios of the expansions and contractions in $E^{u}$ and $E^{s}$ as compared to $E^{c}$. If $\left.T f\right|_{E^{c}}$ is close to conformal the center bunching conditions are satisfied. ${ }^{\ddagger}$

We say that $f$ is BW partially hyperbolic and center bunched, if it satisfies the Burns-Wilkinson conditions.

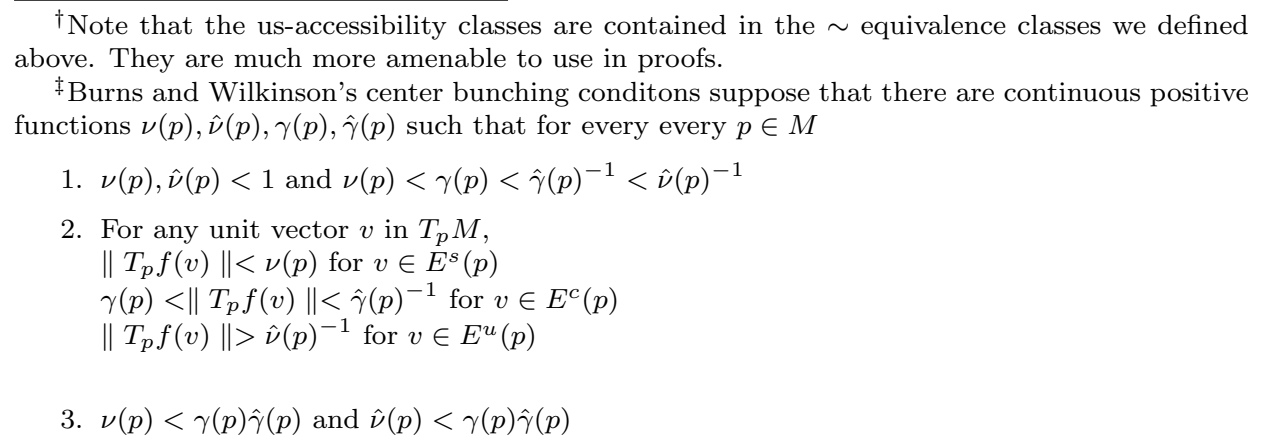

The second condition is the partial hyperbolicity and the third the center bunching. 
Theorem (Burns-Wilkinson). [BuWi3] Let $f$ be $C^{2}$, volume preserving, BW partially hyperbolic and center bunched and essentially accessible. Then $f$ is ergodic and in fact a K-automorphism.

When the dimension of the center bundle $E^{c}$ is one the bunching conditions are automatically satisfied. So it follows as a simple corollary that:

Corollary (Burns-Wilkinson). Conjecture $A$ is true when dimension $E^{c}$ is one.

Even more is true when the dimension of the center bundle $E^{c}$ is one, Federico and Jana Rodrigues-Hertz, and Raul Ures prove the Main Conjecture.

Theorem ([RHRHUr]). When the dimension of $E^{c}$ is one, Conjecture A, Conjecture $\mathrm{B}$ for volume preserving diffeomorphisms and hence the Main Conjecture all are true.

Towards Conjecture B in general there is [DoWi] in the $C^{1}$ topology.

The major new elements in the proofs of the series of theorems on stable ergodicity of partially hyperbolic systems are dynamically defined sets called juliennes which can be used to estimate Lebesgue volumes either directly or by proving that they form a Lebesgue density basis and an analysis of the stable and unstable holonomy maps which are julienne quasi-conformal.

Partial hyperbolicty and center bunching are easily seen to be open conditions and us-accessibility is frequently easily proven to hold in an open neighborhood of a given example. Sometimes even e-accessibility is (not so easily) proved to hold in the neighborhood of a given example[RH]. The situation is good enough to be able to conclude stable ergodicity in the $C^{2}$ topology of quite a few examples. Here are several examples. See [BuPuShWi, PuSh5] for more details and for more on the current state of affairs.

1 The product of a volume preserving Anosov diffeomorphism and any other other volume preserving diffeomorphism can be arbitrarily $C^{\infty}$ closely approximated by a partially hyperbolic, us-accessible stably ergodic diffeomorphism [ShWi1, BuPuShWi], as long as the hyperbolicity of the Anosov diffeomorphism is strong enough to produce a partially hyperbolic splitting of the tangent bundle. (Conjecturally an open and dense set of perturbations is ergodic.) So the KAM phenomenon seems to be dominated by the hyperbolic phenomenon and ergodicity of weakly coupled systems of KAM and Anosov type should be expected to be ergodic.

2 The time $t$ map of the geodesic flow of a manifold of negative curvature is stably ergodic.

3 Skew products which are compact group extensions over standard Anosov diffeomorphisms are generically us-accessible and $C^{2}$ stably ergodic, $[\mathrm{Br} 1$, $\mathrm{Br} 2, \mathrm{BuWi1}, \mathrm{FiPa}]$.

4 Ergodic toral automorphisms having a two dimensional invariant subspace with isometric derivative and some mild extra technical conditions are $C^{r}$ stably ergodic for a fairly large $r[\mathrm{RH}]$. 
5 Partially hyperbolic affine diffeomorphisms of finite volume compact homogeneous spaces of simple Lie groups are stably ergodic. We discuss these below.

Systems whose Lyapunov exponents are non-zero, called non-uniformly hyperbolic, were introduced by Pesin and play a large role in the ergodic theory of volume preserving diffeomorphisms and the study of SRB measures. Pesin's paper [Pe1] raises the question if in dimension bigger than two those diffeomorphisms without zero Lyapunov exponents are generic. We have mentioned above that KAM theory produces open sets of volume preserving diffeomorphisms with positive measure sets of invariant tori which have no hyperbolicity. So the answer to the question is "no". But it may be an either/or situation.

Problem (4). [ShWi2] Is it true for generic $f \in \operatorname{Diff}_{\mu}^{r}(M)$ that for almost every ergodic component of $f$ either all the Lyapunov exponents of $f$ are 0 or none of the Lyapunov exponents of $f$ are 0 ( $\mu$ - a.e.)?

For some partially hyperbolic diffeomorphisms zero exponents were perturbed away in [ShWi2], which produces pathological center foliations. More of this is carried out in [HiPe] and for the $C^{1}$ topology in [BaBo]. So there is some evidence that the answer to the problem is "yes" at least for stably ergodic or partially hyperbolic diffeomorphisms. The problem is even interesting when restricted to ergodic diffeomorphisms so there is only one ergodic component. When $r=1$, Mañé and Bochi prove for two dimensional manifolds that generically all the exponents are zero or the diffeomorphism is Anosov [Mañ1], [Boc].

3.2. Possible Extensions. How might the Anosov-Hopf argument be transported from the category of volume preserving diffeomorphisms to most of $\operatorname{Diff}^{r}(M)$ ? and especially to the existence of SRB measures? Here we enter a more speculative realm. First we recall the definition of SRB measures and some suggestions from [ShWi2].

Given $f \in \operatorname{Diff}^{r}(M)$ (not necessarily preserving $\mu$ ) a closed $f$ invariant set $A \subset M$ and an $f$ invariant ergodic measure $\nu$ on $A$, we define the basin of $A$ to be the set of points $x \in M$ such that $f^{n}(x) \rightarrow A$ and for every continuous function $\phi: M \rightarrow \mathbb{R}$

$$
\lim _{n \rightarrow \infty} \frac{1}{n}\left(\phi(x)+\cdots+\phi\left(f^{n}(x)\right)\right) \rightarrow \int_{A} \phi(x) d \nu .
$$

$\nu$ is an $S R B$ measure and $A$ an $S R B$ attractor (or an ergodic attractor) if the $\mu$ measure of the basin of $A$ with respect to $\mu$ is positive.

It follows from the definition that a diffeomorphism has at most countably many SRB measures. We can more formally describe the Sinai, Ruelle and Bowen [Si, Ru1, BoRu] result already referred to. If $f$ is a $C^{2}$ Axiom A no cycle diffeomorphism then $\mu$ almost every point in $M$ is in a basin of an SRB measure and there are only finitely many SRB measures. It is this result that one would like to extend into a (more) general context. 
The next problem obviously presents itself from the presentation.

Problem (5). For $r \geq 2$ is it true for the generic $f$ in $\operatorname{Diff}^{r}(M)$ that the union of the basins of the SRB attractors of $f$ has full $\mu$ measure in $M$ ?

This natural question is on the minds of quite a few people. See for example [Ru1, BuPuShWi, Pa, Vi1, You1, You2, BoDíVi]. One way to approach the problem along the lines of the Anosov-Hopf argument and as in [Pe1], [PuSh1] might be via an analogue of the either/or question on Lyapunov exponents for volume preserving diffeomorphisms above. For $r \geq 2$ there is no known analogue without the volume preserving hypothesis of the robust positive measure set of invariant tori with zero Lyapunov exponents which occurs via KAM theory. See [Vi2], [BuPuShWi].

Problem (6). For $r \geq 2$ is it true for the generic $f$ in $\operatorname{Diff}^{r}(M)$ and any weak limit $\nu$ of the push forwards $\frac{1}{n} \sum_{1}^{n} f_{*}^{j} \mu$ that almost every ergodic component of $\nu$ has some exponents not equal to $0(\nu-$ ae)? All exponents not equal to 0 ?

Partially hyperbolic systems are a natural domain to begin considering problems [5] and [6]. When the volume is not preserved and we distinguish future behavior from the past the accessibility equivalence relation has to be adapted. Even for partially hyperbolic $f$ it is not entirely clear how to do this. So suppose $f$ partially hyperbolic . Let $W^{u u}(x)$ and $W^{s s}(x)$ denote the strong unstable and stable manifolds which are known to exist tangent to the $E^{u}$ and $E^{s}$ foliations. For $x, y \in M$ define $x>y$ if $W^{u u}(x) \cap W^{s s}(y) \neq \emptyset$. Transitivize $>$ to a partial order on $M$ and declare $x \sim y$ if $x>y$ and $y>x$. The $\sim$ equivalence classes may play a role similar to us-accessibility classes.

Problem (7). For the generic partially hyperbolic $f$, do all $\sim$ equivalence classes which are minimal with respect to $>$ have SRB measures?

3.3. A little hyperbolicity. Now that we have given a lot of examples, we return to our theme that a little hyperbolicity goes a long way towards ergodicity. We ask how often can we prove that hyperbolicity does exist in the guise of some non-zero Lyapunov exponents. Some specific families of non-uniformly hyperbolic dynamical systems have been worked out which contain positive measure sets in the parameter space with SRB measures having non-zero Lyapunov exponents. Most prominent among these families are the quadratic and Henon families, see [Ja, Ly, Be, BeCa, You1, You2, Vi1]. The proofs are difficult. One would like to find a fairly general principle which guarantees that a family has a positive measure set of parameters which have an SRB measure with a positive Lyapunov exponent.

One attempt posits that rich enough families of dynamical systems should have members with positive Lyapunov exponents. Examples have been constructed with uncertain but evocative results. Let $M$ have a Riemannian metric and let $G$ be a group of isometries of $M$ which is transitive on the projectivized tangent bundle of $M$. Let $\mu$ be the Riemannian volume. Let $f_{\epsilon}$ be a family of $C^{r}$ dynamical systems defined on $M$ depending on $\epsilon$. For fixed $\epsilon$, consider the family $G f_{\epsilon}=\left\{g f_{\epsilon}, g \in G\right\}$. Give $G f_{\epsilon}$ the push forward of the Haar measure on $G$. If $f_{\epsilon}$ preserves $\mu$ let $H(\epsilon)$ 
be the average over $G f_{\epsilon}$ of the entropy of $g f_{\epsilon}$ with respect to $\mu$. This is the case in example 3 below. If $f_{\epsilon}$ does not preserve $\mu$ but $g f_{\epsilon}$ has a unique SRB measure for each $g \in G$, let $H(\epsilon)$ be the average over $G f_{\epsilon}$ of the entropy of $g f_{\epsilon}$ with respect to this SRB measure. This is the case in examples 1 and 2 below. We compare $H(\epsilon)$ to the random Lyapunov exponents with respect to random products of elements of $G f_{\epsilon}$ which we shall call

$$
R(\epsilon)=\int_{\mathbb{P} T M} \ln \left|T f_{\epsilon}(v)\right| d v
$$

where $\mathbb{P} T M$ is the projectivized tangent bundle of $M$. It is usually easy to see that $R(\epsilon)$ is positive. When $H(\epsilon)$ is positive then there are obviously positive measure sets in the parameter space with positive Lyapunov exponents and positive entropy. Here are the results for a few families.

1 Blaschke products [PujRoSh]

The family of dynamical systems does not depend on $\epsilon$; we take $f_{\epsilon}=B$ where

$$
B(z)=\theta_{0} \prod_{i=1}^{n} \frac{z-a_{i}}{1-z \overline{a_{i}}},
$$

$n \geq 2, a_{i} \in \mathbb{C},\left|a_{i}\right|<1, i=1 \ldots n$, and $\theta_{0} \in \mathbb{C}$ with $\left|\theta_{0}\right|=1$.

The group $G$ is the unit circle $\mathbb{T}$ in the complex plane, $\mathbb{C}$. Its elements are denoted by $\theta$. Now we take

$$
\mathbb{T} B=\{\theta B\}_{\{\theta \in \mathbb{T}\}}
$$

Then

$$
H(\epsilon) \geq R(\epsilon)
$$

$(H(\epsilon)$ is always positive)

2 Expanding maps of the circle [LlShSi]

The dynamical systems are $f_{k, \alpha, \epsilon}: \mathbb{S}^{1} \rightarrow \mathbb{S}^{1}$ which when written mod 1 are of the form

$$
f_{k, \alpha, \epsilon}: x \mapsto k x+\alpha+\epsilon \sin (2 \pi x) .
$$

The group is $\mathbb{S}^{1}, \alpha$ ranges over $\mathbb{S}^{1}$ and $k \geq 2$. Then for small $\epsilon$ the average over $\alpha$ of the entropy $H(\epsilon)$ is smaller than $R(\epsilon)$, while the max over $\alpha$ of the entropies of $f_{k, \alpha, \epsilon}$ is larger than $R(\epsilon)$. In the case of the averages the difference is on the order of $\epsilon^{2 k+2} . H(\epsilon)$ is again obviously positive.

3 Twist maps of the sphere [LeShSiWi]

For $\epsilon>0$, we define a one-parameter family of twist maps $f_{\epsilon}$ as follows. Express $\mathbb{S}^{2}$ as the sphere of radius $1 / 2$ centered at $(0,0)$ in $\mathbb{R} \times \mathbb{C}$, so that the coordinates $(r, z) \in \mathbb{S}^{2}$ satisfy the equation

$$
|r|^{2}+|z|^{2}=1 / 4
$$


In these coordinates define a twist map $f_{\epsilon}: \mathbb{S}^{2} \rightarrow \mathbb{S}^{2}$, for $\epsilon>0$, by

$$
f_{\epsilon}(r, z)=(r, \exp (2 \pi i(r+1 / 2) \epsilon) z)
$$

The group is $\mathrm{SO}(3)$. So $\mathrm{SO}(3) f_{\epsilon}=\left\{g f_{\epsilon}, g \in \mathrm{SO}(3)\right\}$.

For small $\epsilon, H(\epsilon)$ seems experimentally to be positive and is provably less than $R(\epsilon)$. $R(\epsilon)$ tends to infinity with $\epsilon$ and experimentally $R(\epsilon)$ and $H(\epsilon)$ are asymptotic. If we add a small fixed amount of randomization $\delta$ to the each $g$ in $g f_{\epsilon}$ and average the Lyapunov exponents of this randomized family over $g \in \mathrm{SO}(3)$, we obtain $R_{\delta}(\epsilon)$ which is indeed asymptotic to $R(\epsilon)$ as $\epsilon \rightarrow \infty$.

4 Linear maps $[\mathrm{DeSh}]$

If, instead of dynamical systems, we consider a linear map $A \in G L(n, \mathbb{C})$ and the family $\mathrm{SU}(n) A$, then the average of the logarithms of the $k$ biggest moduli of eigenvalues of $U A$ over $U \in \mathrm{SU}(n)$ is greater than or equal to the sum of the $k$ largest Lyapunov exponents of random products of matrices from $\mathrm{SU}(n) A$.

There may be a general principle operating here that we haven't put our finger on yet.

\section{Some Differentiable Dynamical Systems}

4.1. Affine diffeomorphisms. The ergodic theory of affine diffeomorphisms of homogeneous spaces has been much studied in its own right, see for example [St1], and contains some of the principal examples studied in smooth dynamics such as the geodesic and horocycle flows on surfaces of constant negative curvature and toral automorphisms. Here we study the question of ergodicity of affine diffeomorphisms in the context of partially hyperbolic dynamical systems with $C^{r}$ perturbations. Our methods of proof recover the stable ergodicity of affine diffeomorphisms when they are stably ergodic among affine perturbations and usually extend this stability to $C^{r}$ perturbations. On this last point there remain some open problems.

Suppose that $G$ is a connected Lie group, $A: G \rightarrow G$ is an automorphism, $B$ is a closed subgroup of $G$ with $A(B)=B, g \in G$ is given, and the affine diffeomorphism

$$
f: G / B \rightarrow G / B
$$

is defined as $f(x B)=g A(x) B$. It is covered by the diffeomorphism

$$
\bar{f}=L_{g} \circ A: G \rightarrow G,
$$

where $L_{g}: G \rightarrow G$ is left multiplication by $g$. 
An affine diffeomorphism $\bar{f}$ induces an automorphism of the Lie algebra $\mathfrak{g}=$ $T_{e} G, \mathfrak{a}(\bar{f})=\operatorname{Ad}_{g} \circ T_{e} A$, where $\operatorname{Ad}_{g}$ is the adjoint action of $g$, and $\mathfrak{g}$ splits into generalized eigenspaces,

$$
\mathfrak{g}=\mathfrak{g}^{u} \oplus \mathfrak{g}^{c} \oplus \mathfrak{g}^{s},
$$

such that the eigenvalues of $\mathfrak{a}(\bar{f})$ are respectively outside, on, or inside the unit circle. These eigenspaces and the direct sums $\mathfrak{g}^{c u}=\mathfrak{g}^{u} \oplus \mathfrak{g}^{c}, \mathfrak{g}^{c s}=\mathfrak{g}^{c} \oplus \mathfrak{g}^{s}$ are Lie subalgebras and hence tangent to connected subgroups $G^{u}, G^{c}, G^{s}, G^{c u}, G^{c s}$.

Proposition ([PuShSt1]). Let $f: G / B \rightarrow G / B$ be an affine diffeomorphism as above such that $G / B$ is compact and supports a smooth $G$-invariant volume. Let $G^{*}$ be any of the groups $G^{u}, G^{c}, G^{s}, G^{c u}, G^{c s}$. Then the orbits of the left $G^{*}$ action on $G / B$ foliate $G / B$. Moreover, $f$ exponentially expands the $G^{u}$-leaves, exponentially contracts the $G^{s}$-leaves, and affects the $G^{c}$-leaves subexponentially.

Now we characterize partial hyperbolicity, bunching and accessibility in the context of affine diffeomorphisms. Let $\mathfrak{h}$ denote the smallest Lie subalgebra of $\mathfrak{g}$ containing $\mathfrak{g}^{\mathfrak{u}} \cup \mathfrak{g}^{\mathfrak{s}}$. It is not hard to see that $\mathfrak{h}$ is an ideal in $\mathfrak{g}$. We call it the hyperbolic Lie subalgebra of $\bar{f}$, and we denote by $H$ the connected subgroup of $G$ tangent to $\mathfrak{h}$, calling it the hyperbolic subroup of $\bar{f}$. Finally, let $\mathfrak{b}$ denote the Lie algebra of $B, \mathfrak{b} \subset \mathfrak{g}$.

Theorem ( $[\mathrm{PuSh} 4])$. Let $f: G / B \rightarrow G / B$ be an affine diffeomorphism as above such that $G / B$ is compact and supports a smooth $G$-invariant volume. Then

(a) $f$ is partially hyperbolic if and only if the hyperbolic Lie subalgebra of $\bar{f}$ is not contained in the Lie algebra of $B, \mathfrak{h} \not \subset \mathfrak{b}$.

(b) If $f$ is partially hyperbolic then it is center bunched.

(c) $f$ has the us-accessibility property if and only if $\mathfrak{g}=\mathfrak{b}+\mathfrak{h}$.

(d) $f$ has the e-accessibility property if and only if $\overline{H B}=G$.

When the stable and unstable foliations are smooth, as in the affine case, usaccessibility is stable. Thus we have:

Theorem. [PuSh4] Let $f: G / B \rightarrow G / B$ be an affine diffeomorphism as above such that $G / B$ is compact and supports a smooth $G$-invariant volume. Then $f$ is stably ergodic among $C^{2}$ volume preserving diffeomorphisms of $G / B$ if (merely) the hyperbolic Lie subalgebra $\mathfrak{h}$ is large enough that $\mathfrak{g}=\mathfrak{b}+\mathfrak{h}$.

If $G$ is simple then any nontrivial $\mathfrak{h}$ is large enough since it is an ideal.

Suppose that $A \in \mathrm{SL}(n, \mathbb{R})$ has some eigenvalues that are not of modulus one, and suppose that $\Gamma$ is a uniform discrete $A$-invariant subgroup of $\operatorname{SL}(n, \mathbb{R})$. Set $M=\mathrm{SL}(n, \mathbb{R}) / \Gamma$. Then left multiplication by $A, L_{A}: M \rightarrow M$, is stably ergodic in $\operatorname{Diff}_{\mu}^{2}(M)$. The case where $n$ is large and all but two eigenvalues have modulus one is interesting, in that the dimension of $G^{u}$ and $G^{s}$ is $n-1$ while the dimension of $G^{c}$ is $(n-1)^{2}$, so the dimension of $G^{c}$ is much larger than that of $G^{u}$ and $G^{s}$. 
At the other extreme are abelian groups. If $G=\mathbb{R}^{n}$ and $B=\mathbb{Z}^{n}$ then translations on the torus, $\mathbb{T}^{n}=\mathbb{R}^{n} / \mathbb{Z}^{n}$ are ergodic if the entries of the element defining the translation are rationally independent, but they are never stably ergodic. An automorphism $A$ of $\mathbb{T}^{n}$ is ergodic if and only if $A$ has no eigenvalues that are roots of unity. A little bit of algebra quickly shows that the hypothesis that $A$ has no eigenvalues which are roots of unity is equivalent to the hypothesis that $\overline{H \mathbb{Z}^{n}}=\mathbb{R}^{n}$ where $H$ is the hyperbolically generated subgroup of $\mathbb{R}^{n}$.

We have concentrated on the accessibility condition because accessibility is a topological property and as such it is not difficult to stipulate easily verifiable conditions which guarantee that it persists under small perturbations.

In a recent remarkable paper, Federico Rodriguez Hertz gives the first examples of a stably e-accessible diffeomorphisms that are not us-accessible, $[\mathrm{RH}]$. They are ergodic, non-hyperbolic diffeomorphisms of tori. The first such occurs in dimension four.

Hertz sometimes uses a technical assumption on the automorphism $A$, which will refer to as the Hertz condition, namely that the characteristic polynomial of $A$ is irreducible over the integers and it can not be written as a polynomial in $t^{k}$, $k \geq 2$.

Theorem. $[\mathrm{RH}]$ Let $A$ be an ergodic toral automorphism of $\mathbb{T}^{n}$.

(a) If $n \leq 5$ then $A$ is stably ergodic in $\operatorname{Diff}_{\mu}^{22}\left(\mathbb{T}^{n}\right)$.

(b) If $n \geq 6, E^{c}$ is two-dimensional, and $A$ satisfies the Hertz condition then $A$ is stably ergodic in $\operatorname{Diff}^{5}\left(\mathbb{T}^{n}\right)$.

The differentiability degrees 22 and 5 are not misprints.

Part of Hertz' proof involves an alternative. Either the perturbation is usaccessible or the stable and unstable manifold foliations are differentiably conjugate to the foliations of the linear example and hence the perturbation has the e-accessibility property.

Problem (8). Is every ergodic toral automorphism stably ergodic in the $C^{r}$ topology for some $r$ ?

The next result is an approximate solution of this problem.

Theorem ([ShWi1]). Every ergodic toral automorphism of $\mathbb{T}^{n}$ that is an isometry on the center bundle $E^{c}$ can be approximated arbitrarily well in Diff ${ }_{\mu}^{\infty}\left(\mathbb{T}^{n}\right)$ by a stably us-accessible, stably ergodic diffeomorphism.

Further examples of partially hyperbolic stably ergodic diffeomorphisms are considered in [BuPuShWi]. These include skew products, frame flows, and Anosovlike diffeomorphisms.

The next theorem shows that the condition for stable ergodicity of affine diffeomorphisms among perturbations which are restricted to be left multiplication by group elements near the identity is the same as e-accessibility. Hence, the julienne 
proof of stable ergodoicity applies to prove the stable ergodicity of these affine diffeomorphisms among affine perturbations as well. This phenomenon is discussed in [PuShSt2].

Theorem ([St2]). Suppose that $f: G / B \rightarrow G / B$ is an affine diffeomorphism such that $M=G / B$ is compact and supports a smooth $G$-invariant volume. Then the following are equivalent.

(a) $f$ is stably ergodic under perturbation by left translations.

(b) $\overline{H B}=G$ where $H$ is the hyperbolically generated subgroup of $G$.

Corollary. Suppose that $f: G / B \rightarrow G / B$ is an affine diffeomorphism such that $M=G / B$ is compact and supports a smooth $G$-invariant volume. Assume that $G$ is simple. Then stable ergodicity of $f$ with respect to perturbation by left translations is equivalent to stable ergodicity among $C^{2}$ volume preserving perturbations.

This corollary and the result of Rodriguez-Hertz naturally lead to a generalization of Problem 10.

Problem (9). For an affine diffeomorphism $f$ of a compact, finite volume $G / B$, is stable ergodicity of $f$ with respect to perturbation by left translations equivalent to stable ergodicity among $C^{2}$ volume preserving perturbations?

We end our discussion of partially hyperbolic diffeomorphisms with a question from $[\mathrm{BuPuShWi}]$ of a very different nature. We have used both the strong unstable and strong stable foliations in our proof of ergodicity, but we don't know an example where this is strictly necessary.

Problem (10). For a partially hyperbolic $C^{2}$ ergodic diffeomorphism $f$ with the e-accessibility property, are the unstable and stable foliations already ergodic and uniquely ergodic?

Unique ergodicity of for horocycle flows was proved by Furstenberg [Fu]. Bowen and Marcus [BoMa] proved the unique ergodicity of the strong stable and unstable manifold foliations in the case where $f$ is the time-one map of a hyperbolic flow. Rodriguez Hertz' result adds more cases in which the invariant foliations are uniquely ergodic, namely those in which they are differentiably conjugate to the invariant foliations of a linear ergodic toral automorphism. Starkov [PuShSt2] proves that unique ergodicity of the strong stable or unstable foliations for all affine diffeomorphisms which are stably ergodic under perturbation by left translation.

In the topological category Bonatti, Díaz, and Ures [BoDíUr] prove the minimality of the stable and unstable foliations for an open and dense set of robustly transitive diffeomorphisms.

4.2. Models. Two dynamical systems $f: M \rightarrow M$ and $g: N \rightarrow N$ are topologically conjugate if there is a homeomorphism $h: M \rightarrow N$ such that $h f=g h$. The dynamical system $f$ is structurally stable if there is a $C^{r}$ neighborhood of $f$ 
such that every $g$ in $U$ is topologically conjugate to $f . .^{\S}$ By the work of Smale, Palis $[\mathrm{PaSm}]$, Robbin [Ro] and Robinson [Rob], diffeomorphisms that satisfy Smale's Axiom A and the strong transversality condition are structurally stable. Mañé [Mañ2] in general and Liao [Li] also in dimension 2 prove that in the $C^{1}$ topology this condition is also necessary. The $C^{2}$ Axiom A strong transversality diffeomorphisms also have finitely many attractors which have SRB measures. These Axiom A strong transversality diffeomorphisms are extraordinarily appealing since they have all the properties we hope for. They are fairly well understood. Yet there remain interesting questions about them. Some of the issues are discussed in [Su]. I will denote the set of Axiom A strong transversality diffeomorphisms of $M$ by $\operatorname{AS}^{r}(M) \cdot$

Since topological entropy is a topological conjugacy invariant and $C^{\infty}$ is dense in $C^{r}$ the Entropy Inequality holds for all $C^{r}$ structurally stable diffeomorphisms. How sharp is the Entropy Inequality as a lower bound for the entropy of dynamical systems in $\operatorname{AS}^{r}(M)$ ? Smale [Sm3] proved that every isotopy class of diffeomorphisms contains an element of $\operatorname{AS}^{r}(M)$. Since the fundamental group can contribute information about the entropy not readable in the homology groups, we restrict ourselves to simply connected manifolds."

Problem (11). Let $M$ be simply connected. Let $I$ be an isotopy class of diffeomorphisms of $M$. Is there a sequence of diffeomorphisms, $f_{n} \in I \cap \operatorname{AS}^{r}(M)$ such that $h\left(f_{n}\right) \rightarrow \ln \left(s\left(f_{*}\right)\right)$ ?

If the restriction that the diffeomorphism lie in $\operatorname{AS}^{r}(M)$ is removed then it is even unknown whether equality may be achieved in the Entropy Inequality within every isotopy class of diffeomorphisms. There are examples where equality may not be achieved with elements of $\operatorname{AS}^{r}(M)$. A diffeomorphism in $\operatorname{AS}^{r}(M)$ with zero entropy is necessarily Morse-Smale. As a result of [ShSu2, FrSh] and [Le], it is known that there are isotopy classes of diffeomorphisms of simply connected manifolds for which $\ln \left(s\left(f_{*}\right)\right)=0$, yet there is no Morse-Smale diffeomorphism in the class. Are there diffeomorphisms in $\operatorname{AS}^{r}(M)$ with arbitrarily small topological entropy in these classes? If not, what is a lower bound on the entropy?

Model elements of $\operatorname{AS}^{r}(M)$ are constructed in every isotopy class of diffeomorphisms in [ShSu2, Fr2, Mal1] from information on chain complexes for $M$ and chain complex endomorphisms induced by $f$. This work is closely related to Smale's work on the structure of manifolds. See also [Sh2, Sh3] for more discussion of this point. There are further relations between stability and homology theory established in [ShWil, RuSu] where the entropy conjecture was first proven for $C^{1}$ diffeomorphisms satisfying Smale's axioms. This work is also related to our next problem.

To close our discussion of structurally stable diffeomorphisms, I recall one other outstanding problem.

\footnotetext{
$\S$ We restrict ourselves to dynamical systems in $\operatorname{Diff}^{r}(M)$ even though the same concepts apply in $\operatorname{End}^{r}(M)$ and to structural stability as opposed to Omega stability for the sake of simplicity of exposition.

ब AS is a fortuitous selection of letters since Anosov, Sinai, Smale, Axiom A and Strong all begin with $\mathrm{A}$ and $\mathrm{S}$.

II See Maller [Mal1, Mal2] for non-simply connected manifolds.
} 
Problem (12). Are all Anosov diffeomorphisms infra-nil?

Smale[Sm2], considered the nil-manifold setting for Anosov diffeomorphisms which was later extended by example [Sh1] to infra-nil manifolds where the corresponding examples of expanding maps were considered. All expanding maps are infra-nil by the results of [Sh1], [Fr1] and Gromov [Gro1] on groups of polynomial growth. For Anosov diffeomorphisms defined on a manifold $M$, it is known that if $M$ is an infra-nil manifold then the diffeomorphism is conjugate to an affine example, [Ma2]. It is not known if all manifolds $M$ supporting Anosov diffeomorphisms are infra-nil manifolds. If one of the bundles $E^{s}$ or $E^{u}$ is one dimensional then problem is answered in the affirmative by [New2]. Perhaps the best results go back to $[\operatorname{Fr} 1]$.

Questions about the classification of manifolds admitting partially hyperbolic diffeomorphisms are raised in section 20 of [PuSh5].

We end the paper by mentioning a few surveys which go into greater depth on some of the issues we have considered, [Sm2, Fr2, Sh1, Sh3, BuPuShWi, PuSh5, Pe3, BoDíVi].

\section{References}

[An] Anosov, D. V., Geodesic flows on closed Riemannian manifolds of negative curvature, Proc. Steklov. Inst. Math. 90 (1967).

[AnSi] Anosov, D. V. and Ya. G. Sinai, Some smooth ergodic systems, Russian Math. Surveys 22 No.5 (1967), London Math. Soc., 103-167.

[BaBo] Baraviera, A. and Bonatti, C., Removing zero Lyapunov exponents, Ergodic Theory Dynam. Systems 23 (2003), 1655-1670.

[Be] Benedicks, M., Non uniformly hyperbolic dynamics: Hénon maps and related dynamical systems. In Proceedings of the International Congress of Mathematicians, Vol. III (Beijing, 2002), 255-264. Errata Vol. I, 651, Higher Ed. Press, Beijing, 2002.

[BeCa] Benedicks, M. and Carleson, L., The dynamics of the Henon map, Annals of Math. bf 133 (1991), 73-169.

[Boc] Bochi,J. Genericity of zero Lyapunov exponents, Ergodic Theory and Dyanmical Systems 22 (2002) 1667-1696.

[BoDíUr] Bonatti,C., Díaz,L.J., Ures,R.,Minimality of strong stable and unstable foliations for partially hyperbolic diffeomorphisms, J. Inst. Math. Jussieu, 1 (2002), 513541.

[BoDíVi] Bonatti,C., Díaz,L.J., Viana,M., Dynamics Beyond Uniform Hyperbolicity. Encyclopedia of Mathematical Sciences 102, Mathematical Physics III, Springer, 2005.

[Bo] Bowen, R., Entropy and the fundamental group. In The structure of attractors in dynamical systems (Proc. Conf., North Dakota State Univ., Fargo, N.D., 1977), 2129, Lecture Notes in Math., 668, Springer, 1978

[BoMa] Bowen, R. and Marcus, B., Unique ergodicity for horocycle foliations, Israel J. Math. 26 (1977), 43-67.

[BoRu] Bowen, R. and Ruelle, D., The ergodic theoryof Axiom A flows, Inventiones Math. 29 (1975), 181-202. 
[Br1] Brin, M., Topological transitivity of one class of dynamical systems and flows of frames on manifolds of negative curvature, Func. Anal. Appl. 9 (1975), 9-19.

[Br2] Brin, M., The topology of group extensions of C systems, Mat. Zametki 18 (1975), 453-465.

[BrPe] Brin, M. and Pesin,Ja., Partially hyperbolic dynamical systems, Math. USSR Izvestija 8 (1974), 177-218.

[BuPuShWi] Burns, K., Pugh, C., Shub, M. and Wilkinson, A., Recent results about stable ergodicity, Proc. Symp. Pure Math 69, Amer. Math. Soc. 2001, 327-366.

[BuWi1] Burns, K. and Wilkinson, A., Stable ergodicity of skew products, Ann. Sci. École Norm. Sup. 32 (1999), 859-889.

[BuWi2] Burns, K. and Wilkinson, A., Better center bunching, preprint.

[BuWi3] Burns, K. and Wilkinson, A., On the ergodicity of partially hyperbolic systems, preprint.

[ChSu] Cheng, C.-Q., and Sun, Y.-S., Existence of invariant tori in three dimensional measure-preserving mappings, Celestial Mech. Dynam. Astronom. 47 (1989/90), 275292.

[DeSh] Dedieu, J.-P. and Shub, M., On Random and Mean Exponents for Unitarily Invariant Probability Measures on $G L(n, C)$. In "Geometric Methods in Dynamical Systems (II)-Volume in Honor of Jacob Palis", Asterisque, 287 (2003) 1-18 Soc. Math. De France.

[LlShSi] de la Llave, R., Shub, M. and Simó, C., Entropy estimates for a family of expanding maps of the circle, preprint.

[DoWi] Dolgopyat, D. and Wilkinson, A., Stable accessibility is $C^{1}$ dense. In Asterisque 287 (2003),33-60.

[FaSh] Fathi, A. and Shub,M., Some Dynamics of Pseudo-Anosov Diffeomorphisms, Expose 10, in Travaux de Thurston sur les Surfaces, Asterisque, 66-67, Societe Mathematiques de France, Paris 1979.

[FiPa] Field, M. and Parry, W., Stable ergodicity of skew extensions by compact Lie groups, Topology 38 (1999), 167-187.

[Fr1] Franks, J.M., Anosov Diffeomorphisms, Proc. Symp. Pure Math. 14, Amer. Math. Soc., 1970, 61-93.

[Fr2] Franks, J.M., Homology Theory and Dynamical Systems, Conference Board of the Mathematical Sciences Regional Conference Series in Mathematics 49, Amer. Mat. Soc., 1982 .

[FrSh] Franks, J. and Shub, M., The Existence of Morse-Smale Diffeomorphisms, Topology, 20 (1981), 273-290.

[Fu] Furstenberg, H., The unique ergodicity of the horocycle flow. In Recent advances in topological dynamics (Proc. Conf., Yale Univ., New Haven, Conn., 1972; in honor of Gustav Arnold Hedlund), 95-115. Lecture Notes in Math., 318, Springer, 1973

[GrPuSh] Grayson, M., Pugh, C. and Shub, M., Stably ergodic diffeomorphisms, Ann. Math. 140 (1994), 295-329.

[Gro1] Gromov, M., Groups of polynomial growth and expanding maps, Inst. des Hautes Études Sci. Publ. Math. 53, (1981), 53-73. 
[Gro2] Gromov, M., Entropy, homology and semialgebraic geometry. in Séminaire Bourbaki, Vol. 1985/86 Astrisque No. 145-146 (1987), 5, 225-240

[HiPe] Hirayama, M. and Pesin, Ya., Non-absolutely Continuous Foliations preprint.

[Ho] Hopf, E., Statistik der geodätischen Linien in Mannigfaltigkeiten negativer Krümmung, Ber. Verh. Sächs. Akad. Wiss. Leipzig 91 (1939), 261-304.

[Ja] Jakobson,M., Absolutely continuous invariant measures for one-parameter families of one dimensioanl maps, Comm. Math. Phys. 81 (1981), 39-88.

[Ka] Katok, A. Lyapunov exponents, entropy and periodic orbits for diffeomorphisms, Inst. Hautes tudes Sci. Publ. Math. 51 (1980), 137-173.

[LeShSiWi] Ledrappier, F., Shub, M., Simó, C. and Wilkinson, A.Random versus deterministic exponents in a rich family of diffeomorphisms, J. Statist. Phys. 113 (2003), 85-149.

[Le] Lenstra, H. W., Jr. Grothendieck groups of abelian group rings, J. Pure Appl. Algebra 20 (1981), 173-193.

[Li] Liao, S.T., On the stability conjecture, Chinese Ann. Math. 1 (1980), 9-30.

[Ly] Lyubich, M., Almost every real quadratic map is either regular or stochastic Ann. of Math. (2) 156 (2002), 1-78.

[Mal1] Maller, M., Fitted diffeomorphisms of nonsimply connected manifolds, Topology 19 (1980), 395-410.

[Mal2] Maller, M., Algebraic problems arising from Morse-Smale dynamical systems. In Geometric dynamics (Rio de Janeiro, 1981), 512-521, Lecture Notes in Math., 1007, Springer, 1983.

[Ma1] Manning, A., Topological entropy and the first homology group. In Dynamical systems-Warwick 1974 (Proc. Sympos. Appl. Topology and Dynamical Systems, Univ. Warwick, Coventry, 1973/1974; presented to E. C. Zeeman on his fiftieth birthday), 185-190. Lecture Notes in Math., 468, Springer, 1975

[Ma2] Manning, A., There are no new Anosov diffeomorphisms on tori, Amer. J. Math. 96 (1974), 422-429.

[Mañ1] Mañé, R., Oseledec's theorem from the generic viewpoint, Proc. of Int. Congress of Math. (1983) Warszawa 1269-1276.

[Mañ2] Mañé, R., A proof of the $C^{1}$ stability conjecture. Inst. des Hautes Études Sci. Publ. Math. No. 66, (1988), 161-210.

[MaPr] Marzantowicz,W. and Przytycki,F., Entropy conjecture for continuous maps of nilmanifolds, preprint.

[MiPr] Misiurewicz, M., and Przytycki, F., Topological entropy and degree of smooth mappings, Bull. Acad. Polon. Sci. Sr. Sci. Math. Astronom. Phys 25 (1977), 573-574.

[New1] Newhouse, S.E., Entropy in Smooth Dynamical Systems. In Proceedings of the International Congress of Mathematicians, August 21-29, 1990, Kyoto, Japan (ed. by Ichiro Satake). The Mathematical Society of Japan, Springer-Verlag, 1285-1294.

[New2] Newhouse, S. E. On codimension one Anosov diffeomorphisms, Amer. J. Math. 92 1970, 761-770.

[Pa] Palis, J., A Global View of Dynamics and a Conjecture on the Denseness of Finitude of Attractors. In Astrisque 261, (2000), 339-351. 
[PaSm] Palis, J. and Smale, S., Structural stability theorems, Proc. Symp. Pure Math. 14, Amer.Math.soc., 1970, 223-231.

[Pe1] Pesin, Ya., Characteristic Lyapunov exponents and smooth ergodic theory, Russian Math. Surveys 32 (1977), no. 4(196), 55-112, 287.

[Pe2] Pesin, Ya., Ergodic properties and dimensionlike characteristics of strange attractors that are close to hyperbolic. In Proceedings of the International Congress of Mathematicians, Vol. 1, 2 (Berkeley, Calif., 1986), 1195-1209, Amer. Math. Soc., 1987.

[Pe3] Pesin, Ya.,Lectures on partial hyperbolicity and stable ergodicity, Zurich Lectures in Advanced Mathematics, European Mathematical Society , Zrich, 2004

[PeSi] Pesin, Ya.B. and Sinai, Ya. G., Gibbs measures for partially hyperbolic attractors, Ergod. Th. and Dynam. Sys., 2 (1982), 417-438.

$[\mathrm{Pu}]$ Pugh, C., On the entropy conjecture: a report on conversations among R. Bowen, M. Hirsch, A. Manning, C. Pugh, B. Sanderson, M. Shub, and R. Williams. In Dynamical systems-Warwick 1974 (Proc. Sympos. Appl. Topology and Dynamical Systems, Univ. Warwick, Coventry, 1973/1974; presented to E. C. Zeeman on his fiftieth birthday), 257-261. Lecture Notes in Math., 468, Springer, 1975

[PuSh1] Pugh, C. and Shub, M., Ergodic Attractors Trans. AMS. Vol. 312 (1989) pp. $1-54$.

[PuSh2] Pugh,C. and Shub,M., Stable Ergodicity and Partial Hyperbolicity. In International Conference on Dynamical Systems: Montevideo 1995, a tribute to Ricado Mane, Pitman Research Notes in Mathematics 362 (F. Ledrappier, J. Lewowicz and S. Newhouse eds.), (1997), 182-187.

[PuSh3] Pugh, C. and M. Shub, Stably ergodic dynamical systems and partial hyperbolicity, J. of Complexity 13 (1997), 125-179.

[PuSh4] Pugh, C. and Shub, M., Stable ergodicity and julienne quasiconformality, J. Eur. Math. Soc. 2 (2000), 1-52.

[PuSh5] Pugh, C. and Shub, M., Stable ergodicity, Bull. Amer. Math. Soc.(N.S.) 41 (2004), 1-41.

[PuShSt1] Pugh, C., Shub, M. and Starkov, A., Corrigendum to Stable ergodicity and julienne quasi-conformality, J. Eur. Math. Soc. 2, 1-52 J.Eur.Math.Soc. Vol 6 (2004), 149-151.

[PuShSt2] Pugh, C., Shub, M. and Starkov, A., Unique Ergodicity, Stable Ergodicity and the Mautner Phenomenon for Diffeomorhisms, preprint

[Puj] Pujals, E., Tangent bundles dynamics and its consequences in Li, Ta Tsien (ed.) et al., Proceedings of the international congress of mathematicians, ICM 2002, Beijing, China, August 20-28, 2002. Vol. III: Invited lectures. Beijing: Higher Education Press. 327-338 (2002).

[PujRoSh] Pujals, E., Robert,L. and Shub,M., Expanding maps of the circle rerevisited: Positive Lyapunov exponents in a rich family, preprint.

[Ro] Robbin, J., A structural stability theorem, Annals of Math. (2) 94 (1971), 447-493.

[Rob] Robinson, C., Structural Stability of $C^{1}$ diffeomorphisms, J. Diff. Equ., 22 (1976), 28-73. 
[RH] Rodriguez Hertz, F., Stable Ergodicity of Certain Linear Automorphisms of The Torus, to appear.

[RHRHUr] Rodriguez Hertz, F., Rodriguez Hertz, J. and Ures, R., Partially hyperbolic sytems with $1 D$-center bundle: I.-Stable Ergodicity, preprint

[Ru1] Ruelle, D., A measure associated with Axiom-A attractors, Amer. J. Math., 98 (1976), 619-654.

[Ru2] Ruelle, D., Turbulent Dynamical Systems. In Proceedings International Congress of Mathematicians (Warszawa, 1983), 271-286.

[RuSu] Ruelle, D. and Sullivan, D., Currents, flows and diffeomorphisms, Topology 14 (1975), 319-327.

[RuWi] Ruelle, D. and Wilkinson, A., Absolutely singular dynamical foliations, Comm. Math. Phys. 219 (2001), 481-487.

[Sh1] Shub, M. Endomorphisms of Compact Differentiable Manifolds, Amer. J. Math. XCI(1969), 175-199.

[Sh2] Shub, M., Dynamical Systems, Filtrations and Entropy, Bull. Amer. Math. Soc. 80 (1974), 27-41.

[Sh3] Shub,M., The Geometry and Topology of Dynamical Systems and Algorithms for Numerical Problems. In Proceedings of the 1983 Beijing Symposium on Differential Geometry and Differential Equations (Editor Liao Shantao) Science Press, Beijing, China 1986, 231-260.

[ShSu1] Shub, M. and Sullivan,D., A Remark on the Lefschetz Fixed Point Formula for Differentiable Maps, Topology, 13 (1974), 189-191.

[ShSu2] Shub, M. and Sullivan,D., Homology Theory and Dynamical Systems, Topology, 14 (1975), 109-132.

[ShWi1] Shub, M. and Wilkinson, A., Stably ergodic approximation: two examples, Ergod. Th. and Dyam. Syst. 20, (2000), 875-894.

[ShWi2] Shub, M. and Wilkinson, A., Pathological foliations and removable zero exponents, Invent. Math. 139, (2000), 495-508.

[ShWil] Shub, M. and Williams, R., Entropy and Stability, Topology, 14 (1975), 329-338.

[Si] Sinai, Ya., Gibbs measures in ergodic theory, Russian Math. Surveys 27 (1972), 21-69.

[Sm1] Smale, S. On the structure of manifolds, Amer. J. Math. 84 (1962), 387-399.

[Sm2] Smale, S., Differentiable dynamical systems, Bull. AMS, 73 (1967), 747-817.

[Sm3] Smale,S., Stability and isotopy in discrete dynamical systems. In Dynamical Systems, edited by M. M. Peixoto,, Academic Press, New York (1973).

[St1] Starkov, A.N., Dynamical Systems on Homogeneous Spaces, Translations of Mathematical Monographs, (190) AMS, Providence, RI., 2000.

[St2] Starkov, A.N., Stable ergodicity among left translations, Appendix to Pugh, C. and Shub, M., Stable ergodicity and julienne quasiconformality, J. Eur. Math. Soc. 2 (2000), 1-52.

[Su] Sullivan, D., Inside and Outside Manifolds. In Proceedings of the International Congress of Mathematicians, 1974, Vol (1), 201-208.

[Vi1] Viana, M., Dynamics: a probabilistic and geometric perspective. In Proceedings of the International Congress of Mathematicians, (Berlin, 1998) Vol.I, 1998, 557-578. 
[Vi2] Viana, M., Almost all cocycles over any hyperbolic system have non-vanishing Lyapunov exponents, preprint

[Wi1] Wilkinson, A., Stable ergodicity of the time one map of a geodesic flow, Ph.D. Thesis, University of California at Berkeley (1995).

[Wi2] Wilkinson, A., Stable ergodicity of the time one map of a geodesic flow, Ergod. Th. and Dynam. Syst. 18 (1998), 1545-1588.

[Xi] Xia, Z., Existence of invariant tori in volume-preserving diffeomorphisms, Ergodic Theory Dynam. Systems 12 (1992), 621-631.

[Yoc] Yoccoz, J.-C., Travaux de Herman sur les tores invariants, Séminaire Bourbaki, Vol. 1991/92, Astérisque No. 206 (1992), Exp. No. 754, 4, 311-344.

[Yom] Yomdin, Y., Volume growth and entropy, Israel J. Math. 57 (1987), 285-300.

[You1] Young, L.-S., Ergodic Theory of Attractors. In Proceedings of the International Congress of Mathematicians, (Zurich, 1994), Birkhäuser, 1995, 1230-1237.

[You2] Young, L.-S., What are SRB measures, and which dynamical sysytems have them? preprint, to appear in JSP.

Michael Shub ,Mathematics Department University of Toronto, Bahen Centre 40 St George St Room 6290, Toronto, Ontario M5S 2E4 Canada

E-mail: michael.shub@utoronto.ca 